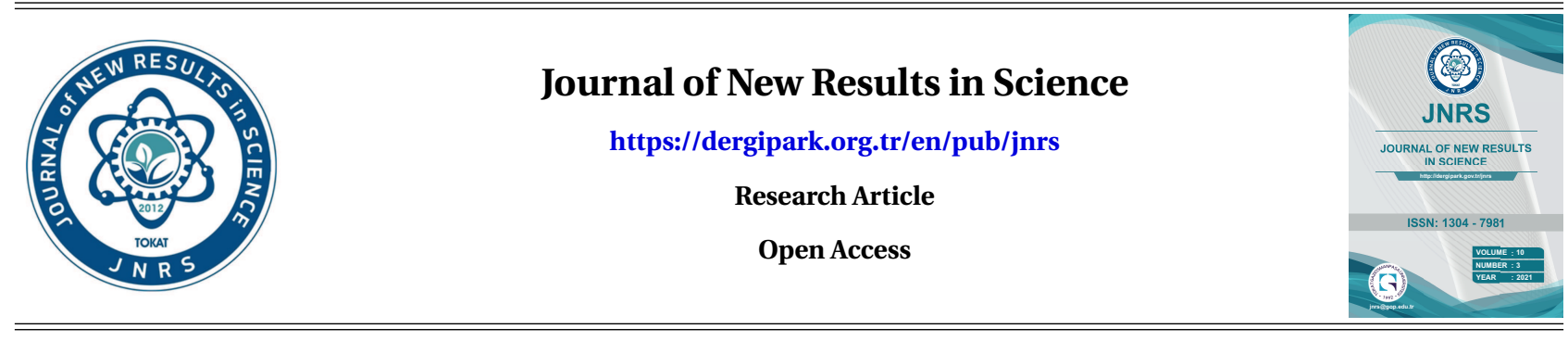

\title{
Local fractional Elzaki transform and its application to local fractional differential equations
}

\author{
Djelloul Ziane $^{1}$ (D) , Mountassir Hamdi Cherif $^{2}$ (D)
}

\author{
Keywords \\ Local fractional \\ calculus, \\ General integral \\ transforms, \\ local fractional \\ differential equations
}

\begin{abstract}
The objective of our work is to couple the Elzaki transform method and the local fractional derivative which is called local fractional Elzaki transform, where we have provided important results of this transformation as local fractional Laplace-Elzaki duality, Elzaki transform of the local fractional derivative and the local fractional integral and the local fractional convolution, also we have presented the properties of some special functions with the local fractional derivative sense. The Elzaki transform was applied to solve some linear local fractional differential equations in order to obtain non-differentiable analytical solutions. The results of the solved examples show the effectiveness of the proposed method.
\end{abstract}

Subject Classification (2020): 44A05, 44A20.

\section{Introduction}

The integral transformations play a crucial role in the resolution of ordinary differential equations, partial differential equations and in the resolution of integral differential equations with integer order or fractional order. They are also widely used by engineers to solve the linear differential equations, the systems of linear partial differential equation, and determine the transfer function of a linear system.

The Laplace transform method [1], the Fourier transform method [2], the Hankel transform method [3], and the Mellin transform method [4] are among the most well-known transformations. Other transformations that have recently appeared include for example the Sumudu transform method [5], the Natural transform method [6], the Elzaki transform method [7-10], the Aboodh transform method [11], the ZZ-transform method [12], the Shehu transform method [13], and others.

Tarig M. Elzaki et al. developed the Elzaki transform method in 2011 from the classical Fourier integral [7], based on the Elzaki transform's mathematical simplicity and fundamental features. This transform has been used by many researchers to facilitate the process of solving the ordinary differential equations without and with variable coefficients [14-17], the higher order differential equations [18], the integro-differential

\footnotetext{
${ }^{1}$ djeloulz@yahoo.com; ${ }^{2}$ mountassir27@yahoo.fr(Corresponding Author)

${ }^{1}$ Faculty of exact sciences and informatics, pole of Ouled Fares, Hassiba Benbouali University of Chlef, Algeria.

${ }^{2}$ Laboratory of complex systems, Oran's Hight School of Electrical and Energetics Engineering (ESGEE-Oran), Oran, Algeria.

${ }^{1,2}$ Laboratory of mathematics and its applications (LAMAP), university of Oran1, Oran, Algeria.

Article History: Received: 26 Jul 2021 - Accepted: 06 Dec 2021 - Published: 31 Dec 2021
} 
equations [19-22] and to the partial differential equations [23-27] in the time domain. Several works have been made by using the Elzaki transform method and its combination with other methods to solve the linear and nonlinear differential equations with the concept of fractional operator, for example the fractional order differential equation arising in RLC electrical circuit, the fractional Porous Medium equation, the fractional higher dimensional initial boundary value problems [28-32].

In this work, we will based on the notion of local fractional operator by presented some important results and properties of the local fractional Elzaki transform and we will extend and applied it to solve the linear differential equations with local fractional derivative. We supported our work, with illustrative examples showing how to apply this transformation to the differential equations on Cantor sets.

\section{Basic of local fractional calculus}

We present the basic definitions and theorems of local fractional derivative, local fractional integral, local fractional Taylor's series and local fractional Laplace transform method.

Definition 2.1. [33-35] Let $f(x) \in C_{\sigma}(a, b)$, so

$$
\left|f(x)-f\left(x_{0}\right)\right|<\epsilon^{\sigma}, \quad 0<\sigma \leqslant 1,
$$

with $\left|x-x_{0}\right|<\delta$, for $\epsilon, \delta \in \mathbb{R}_{+}^{*} . f(x)$ is local fractional continuous.

Definition 2.2. [33-35] The local fractional derivative of $f(x)$ of order $\sigma$ at $x=x_{0}$ is

$$
f^{(\sigma)}(x)=\left.\frac{d^{\sigma} f}{d x^{\sigma}}\right|_{x=x_{0}}=\lim _{x \rightarrow x_{0}} \frac{\Delta^{\sigma}\left(f(x)-f\left(x_{0}\right)\right)}{\left(x-x_{0}\right)^{\sigma}}, 0<\sigma \leqslant 1
$$

where

$$
\Delta^{\sigma}\left(f(x)-f\left(x_{0}\right)\right) \cong \Gamma(1+\sigma)\left[\left(f(x)-f\left(x_{0}\right)\right)\right] .
$$

The local fractional partial differential operator of order $\sigma$ was given by

$$
\frac{\partial^{\sigma} w\left(x_{0}, t\right)}{\partial t^{\sigma}}=\lim _{x \rightarrow x_{0}} \frac{\Delta^{\sigma}\left(w\left(x_{0}, t\right)-w\left(x_{0}, t_{0}\right)\right)}{\left(t-t_{0}\right)^{\sigma}}
$$

where

$$
\left.\Delta^{\sigma}\left(u\left(x_{0}, t\right)-u\left(x_{0}, t_{0}\right)\right) \cong \Gamma(1+\sigma)\left[u\left(x_{0}, t\right)-u\left(x_{0}, t_{0}\right)\right)\right] .
$$

Definition 2.3. [33-35] The local fractional integral of $f(x)$ of order $\sigma$ is

$$
\begin{aligned}
{ }_{a} I_{b}^{(\sigma)} f(x) & =\frac{1}{\Gamma(1+\sigma)} \int_{a}^{b} f(t)(d t)^{\sigma} \\
& =\frac{1}{\Gamma(1+\sigma)} \lim _{\Delta t \rightarrow 0} \sum_{j=0}^{N-1} f\left(t_{j}\right)\left(\Delta t_{j}\right)^{\sigma}
\end{aligned}
$$

where $\Delta t_{j}=t_{j+1}-t_{j}, \Delta t=\max \left\{\Delta t_{0}, \Delta t_{1}, \Delta t_{2}, \cdots\right\}$ and $\left[t_{j}, t_{j+1}\right], t_{0}=a, t_{N}=b$, is a partition of the interval $[a, b]$. 
Definition 2.4. [35-37] The local fractional Laplace transform of $f(x)$ of order $\sigma$ is given by

$$
L_{\sigma}\{f(x)\}=F_{\sigma}(s)=\frac{1}{\Gamma(1+\sigma)} \int_{0}^{\infty} E_{\sigma}\left(-s^{\sigma} x^{\sigma}\right) f(x)(d x)^{\sigma}
$$

If $L F L_{\sigma}\{f(x)\}=F_{\sigma}(s)$, so the inverse formula of (2.7) is

$$
f(x)=L_{\sigma}^{-1}\left\{F_{\sigma}(s)\right\}=\frac{1}{(2 \pi)^{\sigma}} \int_{\beta-i \infty}^{\beta+i \infty} E_{\sigma}\left(s^{\sigma} x^{\sigma}\right) F_{\sigma}(s)(d s)^{\sigma},
$$

$s^{\sigma}=\beta^{\sigma}+i^{\sigma} \infty^{\sigma}, i^{\sigma}$ is the fractal imaginary unit and $\operatorname{Re}(s)=\beta>0$.

Theorem 2.5. [34] If $L_{\sigma}\{f(x)\}=F_{\sigma}(s)$, then one has

$$
L_{\sigma}\left\{f^{(\sigma)}(x)\right\}=s^{\sigma} L_{\sigma}\{f(x)\}-f(0)
$$

\section{Proof.}

see [34]

Theorem 2.6. [34] If $L_{\sigma}\{f(x)\}=F_{\sigma}(s)$, so

$$
L_{\sigma}\left\{{ }_{0} I_{x}^{\sigma} f(x)\right\}=\frac{1}{s^{\sigma}} L_{\sigma}\{f(x)\}
$$

\section{Proof.}

see [34]

Theorem 2.7. [34] If $L_{\sigma}\{f(x)\}=F_{\sigma}(s)$ and $L_{\sigma}\{g(x)\}=G_{\sigma}(s)$, then one has

$$
L_{\sigma}\{f(x) * g(x)\}=F_{\sigma}(z) G_{\sigma}(z)
$$

where

$$
f(x) * g(x)=\frac{1}{\Gamma(1+\sigma)} \int_{0}^{\infty} f(t) g(x-t)(d t)^{\sigma} .
$$

\section{Proof.}

see [34]

Theorem 2.8. [38] Let $f(x) \in C_{\sigma}[a, b]$, so there is a function

$$
\Pi(x)={ }_{a} I_{x}^{(\sigma)} f(x)
$$

its derivative with respect to $(d x)^{\rho}$ is

$$
\frac{d^{\sigma} \Pi(x)}{(d x)^{\sigma}}=f(x), a \leqslant x \leqslant b
$$

\section{Proof.}

see [38] 


\section{Main Result}

Now, we present the local fractional Elzaki transform method (LFET) and some properties are discussed. If there is a new transform operator $L F E_{\sigma}: f(x) \longrightarrow T_{\sigma}(v)$, namely

$$
\operatorname{LFE}_{\sigma}\{f(x)\}=L F E_{\sigma}\left\{\sum_{k=0}^{\infty} a_{k} x^{k \sigma}\right\}=\sum_{k=0}^{\infty} \Gamma(1+k \sigma) a_{k} v^{k \sigma+2 \sigma}
$$

For example if $f(x)=E_{\sigma}\left(i^{\sigma} x^{\sigma}\right)$, we obtain

$$
\begin{aligned}
\operatorname{LFE}_{\sigma}\left\{E_{\sigma}\left(i^{\sigma} x^{\sigma}\right)\right\} & =\operatorname{LFE}\left\{\left\{\sum_{k=0}^{\infty} \frac{i^{k \sigma} x^{k \sigma}}{\Gamma(1+k \sigma)}\right\}\right. \\
& =\sum_{k=0}^{\infty} i^{k \sigma} v^{k \sigma+2 \sigma}
\end{aligned}
$$

and if $f(x)=\frac{x^{\sigma}}{\Gamma(1+\sigma)}$, we get

$$
\operatorname{LFE}_{\sigma}\left\{\frac{x^{\sigma}}{\Gamma(1+\sigma)}\right\}=v^{3 \sigma}
$$

These results can be generalized by this definition.

Definition 3.1. The local fractional Elzaki transform of $f(x)$ of order $\sigma$ is defined as

$$
L F E_{\sigma}\{f(x)\}=T_{\sigma}(\nu)=\frac{1}{\Gamma(1+\sigma)} v^{\sigma} \int_{0}^{\infty} E_{\sigma}\left(-\frac{x^{\sigma}}{v^{\sigma}}\right) f(x)(d x)^{\sigma}, 0<\sigma \leqslant 1
$$

The inverse transformation can be obtained as follows

$$
\operatorname{LFE}_{\sigma}^{-1}\left\{T_{\sigma}(\nu)\right\}=f(x) .
$$

Theorem 3.2. (linearity).

If $L F E_{\sigma}\{f(x)\}=F_{\sigma}(\nu)$ and $L F E_{\sigma}\{g(x)\}=G_{\sigma}(\nu)$, then one has

$$
L F E_{\sigma}\{\lambda f(x)+\mu g(x)\}=\lambda F_{\sigma}(\nu)+\mu G_{\sigma}(\nu)
$$

where $\lambda$ and $\mu$ are constant.

Proof.

Using formula (3.5), we obtain 


$$
\begin{aligned}
\operatorname{LFE}_{\sigma}\{\lambda f(x)+\mu g(x)\} & =\frac{v^{\sigma}}{\Gamma(1+\sigma)} \int_{0}^{\infty} E_{\sigma}\left(-\frac{x^{\sigma}}{v^{\sigma}}\right)\{\lambda f(x)+\mu g(x)\}(d x)^{\sigma} \\
& =\frac{v^{\sigma}}{\Gamma(1+\sigma)} \int_{0}^{\infty}\left[E_{\sigma}\left(-\frac{x^{\sigma}}{v^{\sigma}}\right)(\lambda f(x))+E_{\sigma}\left(-\frac{x^{\sigma}}{v^{\sigma}}\right)(\mu g(x))\right](d x)^{\sigma} \\
& =\lambda \frac{v^{\sigma}}{\Gamma(1+\sigma)} \int_{0}^{\infty} E_{\sigma}\left(-\frac{x^{\sigma}}{v^{\sigma}}\right) f(x)(d x)^{\sigma}+\mu \frac{v^{\sigma}}{\Gamma(1+\sigma)} \int_{0}^{\infty} E_{\sigma}\left(-\frac{x^{\sigma}}{v^{\sigma}}\right) g(x)(d x)^{\sigma} \\
& =\lambda L F E_{\sigma}\{f(x)\}+\mu L F E_{\sigma}\{g(x)\} .
\end{aligned}
$$

This ends the proof.

Theorem 3.3. (local fractional Elzaki-Laplace and Laplace-Elzaki duality).

If $L_{\sigma}\{f(x)\}=F_{\sigma}(s)$ and $L F E_{\sigma}\{g(x)\}=T_{\sigma}(v)$, then one has

$$
\begin{gathered}
\operatorname{LFE}_{\sigma}\{f(x)\}=v^{\sigma} F_{\sigma}\left(\frac{1}{v}\right) . \\
L_{\sigma}\{f(x)\}=s^{\sigma} T_{\sigma}\left(\frac{1}{s}\right) .
\end{gathered}
$$

\section{Proof.}

We show the formula (3.8). Using the formula (3.5) gives

$$
\begin{aligned}
\operatorname{LFE}_{\sigma}\{f(x)\} & =v^{\sigma} \frac{1}{\Gamma(1+\alpha)} \int_{0}^{\infty} E_{\sigma}\left(-\frac{x^{\sigma}}{v^{\sigma}}\right) f(x)(d x)^{\sigma} \\
& =v^{\sigma} \frac{1}{\Gamma(1+\sigma)} \int_{0}^{\infty} E_{\sigma}\left(-\left(\frac{1}{v}\right)^{\sigma} x^{\sigma}\right) f(x)(d x)^{\sigma} \\
& =v^{\sigma} F_{\sigma}\left(\frac{1}{v}\right)
\end{aligned}
$$

Proof of the formula (3.9). We have

$$
T_{\sigma}(v)=v^{\sigma} F_{\sigma}\left(\frac{1}{v}\right)
$$

By substituting $v=\frac{1}{s}$, we obtain

$$
T_{\sigma}\left(\frac{1}{s}\right)=\frac{1}{s^{\sigma}} F_{\sigma}(s)
$$

then

$$
F_{\sigma}(s)=s^{\sigma} T_{\sigma}\left(\frac{1}{s}\right)
$$

therefore, we get

$$
L_{\sigma}\{f(x)\}=s^{\sigma} T_{\sigma}\left(\frac{1}{s}\right)
$$

This ends the proof. 
Theorem 3.4. (Elzaki transform of local fractional derivative).

If $L F E_{\sigma}\{f(x)\}=T_{\sigma}(v)$, so

$$
\operatorname{LFE}_{\sigma}\left\{D_{0+}^{\sigma} f(x)\right\}=\frac{1}{v^{\sigma}} T_{\sigma}(v)-v^{\sigma} f(0), 0<\sigma \leqslant 1
$$

and

$$
L F E_{\sigma}\left\{D_{0+}^{n \sigma} f(x)\right\}=\frac{1}{v^{n \sigma}} T_{\sigma}(\nu)-\sum_{k=0}^{n-1} v^{(k-n+2) \sigma} f^{(k \sigma)}(0), 0<\sigma \leqslant 1 .
$$

\section{Proof.}

We proof the formula (3.10). Using the formula (3.8) and the integration by parts [41], we get the following

$$
\begin{aligned}
\operatorname{LFE}_{\sigma}\left\{f^{(\sigma)}(x)\right\} & =v^{\sigma} F_{\sigma}\left(\frac{1}{v}\right)=\frac{v^{\sigma}}{\Gamma(1+\sigma)} \int_{0}^{\infty} E_{\sigma}\left(-\frac{x^{\sigma}}{v^{\sigma}}\right) f^{(\sigma)}(x)(d x)^{\sigma} \\
& =\frac{v^{\sigma}}{\Gamma(1+\sigma)}\left([-\Gamma(1+\sigma) f(0)]+\frac{1}{v^{\sigma}} \lim _{t \longrightarrow \infty} \int_{0}^{t} E_{\sigma}\left(-\frac{x^{\sigma}}{v^{\sigma}}\right) f(x)(d x)^{\sigma}\right) \\
& =-v^{\sigma} f(0)+\frac{1}{v^{\sigma}}\left(\frac{v^{\sigma}}{\Gamma(1+\sigma)} \int_{0}^{\infty} E_{\sigma}\left(-\frac{x^{\sigma}}{v^{\sigma}}\right) f(x)(d x)^{\sigma}\right) \\
& =\frac{1}{v^{\sigma}} T_{\sigma}(v)-v^{\sigma} f(0) .
\end{aligned}
$$

To demonstrate the validity of (3.11), we use the mathematical induction.

If $n=1$ and according to (3.11), we have

$$
\operatorname{LFE}_{\sigma}\left\{D_{0+}^{\sigma} f(x)\right\}=\frac{1}{v^{\sigma}} T_{\sigma}(\nu)-v^{\sigma} f(0),
$$

so, according to (3.10), we note that the formula holds when $n=1$.

Assume inductively that the formula holds for $n$, we get

$$
L F E_{\sigma}\left\{D_{0+}^{n \sigma} f(x)\right\}=\frac{1}{v^{n \sigma}} T_{\sigma}(v)-\sum_{k=0}^{n-1} v^{(k-n+2) \sigma} f^{(k \sigma)}(0) .
$$

It remains to show that (3.11) is true for $n+1$. Let $D_{0+}^{n \sigma} f(x)=g(x)$ and according to (3.10) and (3.12), we have

$$
\begin{aligned}
L F E_{\sigma}\left[D_{0+}^{(n+1) \sigma} f(x)\right] & =\operatorname{LFE}_{\sigma}\left[D_{0+}^{\sigma} g(x)\right]=\frac{1}{v^{\sigma}} G_{\sigma}(v)-v^{\sigma} g(0) \\
& =\frac{1}{v^{\sigma}}\left[\frac{1}{v^{n \sigma}} T_{\sigma}(v)-\sum_{k=0}^{n-1} v^{(k-n+2) \sigma} f^{(k \sigma)}(0)\right]-v^{\sigma} g(0) \\
& =\frac{1}{v^{(n+1) \sigma}} T_{\sigma}(v)-\sum_{k=0}^{n-1} v^{(k-n+1) \sigma} f^{(k \sigma)}(0)-v^{\sigma} f^{(n \sigma)}(x) \\
& =\frac{1}{v^{(n+1) \sigma}} T_{\sigma}(\nu)-\sum_{k=0}^{n} v^{(k-n+1) \sigma} f^{(k \sigma)}(0) .
\end{aligned}
$$


Therefore the formula (3.11) is true for $n+1$. Thus by the principle of mathematical induction, for all $n \geqslant 1$ the formula (3.11) holds.

Theorem 3.5. (Elzaki transform of local fractional integral ).

If $L F E_{\sigma}\{f(x)\}=T_{\sigma}(\nu)$, so

$$
\operatorname{LFE}_{\alpha}\left\{{ }_{0} I_{x}^{(\sigma)} f(x)\right\}=v^{\sigma} T_{\sigma}(v)
$$

\section{Proof.}

Let $H(x)={ }_{0} I_{x}^{(\sigma)} f(x)$. According to the (theorem 3.2.9, [38]), we get

$$
D_{0+}^{\sigma} H(x)=f(x)
$$

and $H(0)=0$.

Taking the local fractional Elzaki transform on both sides of (3.14), we have

$$
\operatorname{LFE}_{\sigma}\left\{D_{0+}^{\sigma} H(x)\right\}=\operatorname{LFE}_{\sigma}\{f(x)\} .
$$

Which give

$$
\frac{1}{v^{\sigma}} \operatorname{LFE}\{H(x)\}=T_{\sigma}(v)
$$

because $H(0)=0$, and $L F E_{\sigma}\{f(x)\}=T_{\sigma}(v)$.

Thus we get

$$
\operatorname{LFE}_{\sigma}\left\{{ }_{0} I_{x}^{(\sigma)} f(x)\right\}=v^{\sigma} T_{\sigma}(\nu)
$$

Theorem 3.6. (local fractional convolution).

If $L F E_{\sigma}\{f(x)\}=T_{\sigma}(v)$ and $L F E_{\sigma}\{g(x)\}=G_{\sigma}(v)$,

then one has

$$
\operatorname{LFE}_{\sigma}\left\{(f(x) * g(x))_{\sigma}\right\}=\frac{1}{v^{\sigma}} T_{\sigma}(v) G_{\sigma}(v)
$$

where

$$
(f(x) * g(x))_{\sigma}=\frac{1}{\Gamma(1+\sigma)} \int_{0}^{\infty} f(\theta) g(r-\theta)(d r)^{\sigma} .
$$




\section{Proof.}

The Laplace transform of fractional order of the function $(f(x) * g(x))_{\sigma}$ is

$$
L_{\sigma}\left\{(f(x) * g(x))_{\sigma}\right\}=L_{\sigma}\{f(x)\} L_{\sigma}\{g(x)\}
$$

Using the formula (3.8) gives

$$
\begin{aligned}
\operatorname{LFE}_{\sigma}\left\{(f(x) * g(x))_{\sigma}\right\} & =v^{\sigma} L_{\sigma}\{f(x) * g(x)\} \\
& =v^{\sigma} L_{\sigma}\{f(x)\} L_{\sigma}\{g(x)\} \\
& =\frac{1}{v^{\sigma}}\left(v^{\sigma} L_{\sigma}\{f(x)\} v^{\sigma} L_{\sigma}\{g(x)\}\right) \\
& =\frac{1}{v^{\sigma}} T_{\sigma}(v) G_{\sigma}(v) .
\end{aligned}
$$

This completes the proof.

\subsection{Local fractional Elzaki transform of somes special functions}

In the all following results, we relied on the formula (3.5), and some of the results found in the references [39-41].

1) If $f(x)=1$, we get

$$
\begin{aligned}
\operatorname{LFE}_{\sigma}\{1\} & =\frac{1}{\Gamma(1+\sigma)} v^{\sigma} \int_{0}^{\infty} E_{\sigma}\left(-\frac{x^{\sigma}}{v^{\sigma}}\right)(d x)^{\sigma} \\
& =v^{\sigma} \lim _{t \rightarrow \infty}\left[-v^{\sigma} E_{\sigma}\left(-\frac{x^{\sigma}}{v^{\sigma}}\right)\right]_{0}^{t} \\
& =v^{2 \sigma}
\end{aligned}
$$

2) If $f(x)=\frac{x^{\sigma}}{\Gamma(1+\sigma)}(0<\sigma \leqslant 1)$, by using the integration by parts [41], we obtain the following

$$
\begin{aligned}
\operatorname{LFE}_{\sigma}\left\{\frac{x^{\sigma}}{\Gamma(1+\sigma)}\right\} & =\frac{1}{\Gamma(1+\sigma)} v^{\sigma} \int_{0}^{\infty} E_{\sigma}\left(-\frac{x^{\sigma}}{v^{\sigma}}\right) \frac{x^{\sigma}}{\Gamma(1+\sigma)}(d x)^{\sigma} \\
& =\frac{v^{\sigma}}{\Gamma(1+\sigma)} \lim _{t \rightarrow \infty}\left(\int_{0}^{t}\left(-v^{\sigma} E_{\sigma}\left(-\frac{x^{\sigma}}{v^{\sigma}}\right)\right)^{(\sigma)} \frac{x^{\sigma}}{\Gamma(1+\sigma)}(d x)^{\sigma}\right) \\
& =v^{\sigma} \lim _{t \rightarrow \infty}\left(\left[-v^{\sigma} E_{\sigma}\left(-\frac{x^{\sigma}}{v^{\sigma}}\right) \frac{x^{\sigma}}{\Gamma(1+\sigma)}\right]_{0}^{t}+\frac{v^{\sigma}}{\Gamma(1+\sigma)} \int_{0}^{t} E_{\sigma}\left(-\frac{x^{\sigma}}{v^{\sigma}}\right)(d x)^{\sigma}\right) \\
& =\lim _{t \rightarrow \infty}\left(v^{2 \sigma}\left[-v^{\sigma} E_{\sigma}\left(-\frac{x^{\sigma}}{v^{\sigma}}\right)\right]_{0}^{t}\right) .
\end{aligned}
$$

Because $\lim _{t \rightarrow \infty}\left[-v^{\sigma} E_{\sigma}\left(-\frac{x^{\sigma}}{v^{\sigma}}\right) \frac{x^{\sigma}}{\Gamma(1+\sigma)}\right]_{0}^{t}=0$. 
Therefore,

$$
\begin{aligned}
\operatorname{LFE}_{\sigma}\left\{\frac{x^{\sigma}}{\Gamma(1+\sigma)}\right\} & =\lim _{t \rightarrow \infty}\left(v^{2 \sigma}\left[-v^{\sigma} E_{\sigma}\left(-\frac{x^{\sigma}}{v^{\sigma}}\right)\right]_{0}^{t}\right) \\
& =v^{3 \sigma} .
\end{aligned}
$$

3) If $f(x)=E_{\sigma}\left((a x)^{\sigma}\right)$, using the formula (3.5) gives

$$
\begin{aligned}
L F E_{\sigma}\left\{E_{\sigma}\left((a x)^{\sigma}\right)\right\} & =\frac{v^{\sigma}}{\Gamma(1+\sigma)} \int_{0}^{\infty} E_{\sigma}\left(-\frac{x^{\sigma}}{v^{\sigma}}\right) E_{\sigma}\left((a x)^{\sigma}\right)(d x)^{\sigma} \\
& =\frac{v^{\sigma}}{\Gamma(1+\sigma)} \int_{0}^{\infty} E_{\sigma}\left(-\frac{1-(a v)^{\sigma}}{v^{\sigma}} x^{\sigma}\right)(d x)^{\sigma} \\
& =v^{\sigma} \lim _{t \rightarrow \infty}\left[\frac{-v^{\sigma}}{1-(a v)^{\sigma}} E_{\sigma}\left(-\frac{1-(a v)^{\sigma}}{v^{\sigma}} x^{\sigma}\right)\right]_{0}^{t} \\
& =\frac{v^{2 \sigma}}{1-(a v)^{\sigma}}
\end{aligned}
$$

4) If $f(x)=\frac{x^{\sigma}}{\Gamma(1+\sigma)} E_{\sigma}\left((a x)^{\sigma}\right)$, by using the definition, and the integration by parts [41], we have

$$
\begin{aligned}
\operatorname{LFE}_{\sigma}\left\{\frac{x^{\sigma}}{\Gamma(1+\sigma)} E_{\sigma}\left((a x)^{\sigma}\right)\right\}= & \frac{v^{\sigma}}{\Gamma(1+\sigma)} \int_{0}^{\infty} E_{\sigma}\left(-\frac{x^{\sigma}}{v^{\sigma}}\right) \frac{x^{\sigma}}{\Gamma(1+\sigma)} E_{\sigma}\left((a x)^{\sigma}\right)(d x)^{\sigma} \\
= & \frac{v^{\sigma}}{\Gamma(1+\sigma)} \lim _{t \rightarrow \infty} \int_{0}^{t}\left(\frac{-v^{\sigma}}{1-(a v)^{\sigma}} E_{\sigma}\left(-\frac{1-(a v)^{\sigma}}{v^{\sigma}} x^{\sigma}\right)\right)^{(\sigma)} \frac{x^{\sigma}}{\Gamma(1+\sigma)}(d x)^{\sigma} \\
= & v^{\sigma} \lim _{t \rightarrow \infty}\left[\frac{-v^{\sigma}}{1-(a v)^{\sigma}} E_{\sigma}\left(-\frac{1-(a v)^{\sigma}}{v^{\sigma}} x^{\sigma}\right) \frac{x^{\sigma}}{\Gamma(1+\sigma)}\right]_{0}^{t} \\
& +\frac{v^{\sigma}}{\Gamma(1+\sigma)} \lim _{t \rightarrow \infty}^{t} \int_{0}^{t} \frac{v^{\sigma}}{1-(a v)^{\sigma}} E_{\sigma}\left(-\frac{1-(a v)^{\sigma}}{v^{\sigma}} x^{\sigma}\right)(d x)^{\sigma} \\
= & v^{\sigma} \lim _{t \rightarrow \infty}\left[\frac{-v^{2 \sigma}}{\left(1-(a v)^{\sigma}\right)^{2}} E_{\sigma}\left(-\frac{1-(a v)^{\sigma}}{v^{\sigma}} x^{\sigma}\right)\right]_{0}^{t}
\end{aligned}
$$

because $\lim _{t \rightarrow \infty}\left[\frac{-\nu^{\sigma}}{1-(a v)^{\sigma}} E_{\sigma}\left(-\frac{1-(a v)^{\sigma}}{\nu^{\sigma}} x^{\sigma}\right) \frac{x^{\sigma}}{\Gamma(1+\sigma)}\right]_{0}^{t}=0$.

Therefore, we get

$$
\operatorname{LFE}_{\sigma}\left\{\frac{x^{\sigma}}{\Gamma(1+\sigma)} E_{\sigma}\left((a x)^{\sigma}\right)\right\}=\frac{v^{3 \sigma}}{\left(1-(a v)^{\sigma}\right)^{2}} .
$$

5) If $f(x)=\sin _{\sigma}\left((a x)^{\sigma}\right)(0<\sigma \leqslant 1)$, by using the formula (3.5), we get

$$
\begin{aligned}
\operatorname{LFE}_{\sigma}\left\{\sin _{\sigma}\left((a x)^{\sigma}\right)\right\} & =\frac{v^{\sigma}}{\Gamma(1+\sigma)} \int_{0}^{\infty} E_{\sigma}\left(-\frac{x^{\sigma}}{v^{\sigma}}\right) \frac{E_{\sigma}\left(i^{\sigma}(a x)^{\sigma}\right)-E_{\eta}\left(-i^{\sigma}(a x)^{\sigma}\right)}{2 i^{\sigma}}(d x)^{\sigma} \\
& =\frac{1}{2 i^{\sigma}} \frac{v^{\sigma}}{\Gamma(1+\sigma)} \int_{0}^{\infty}\left[E_{\sigma}\left(-\frac{1-(a v)^{\sigma} i^{\sigma}}{v^{\sigma}} x^{\sigma}\right)-E_{\sigma}\left(-\frac{1+(a v)^{\sigma} i^{\sigma}}{v^{\sigma}} x^{\sigma}\right)\right](d x)^{\sigma} \\
& =\frac{v^{\sigma}}{2 i^{\sigma}} \lim _{t \rightarrow \infty}\left[-\frac{v^{\sigma}}{1-(a v)^{\sigma} i^{\sigma}} E_{\sigma}\left(-\frac{1-(a v)^{\sigma} i^{\sigma}}{v^{\sigma}} x^{\sigma}\right)+\frac{v^{\sigma}}{1+(a v)^{\sigma} i^{\sigma}} E_{\sigma}\left(-\frac{1+(a v)^{\sigma} i^{\sigma}}{v^{\sigma}} x^{\sigma}\right)\right]_{0}^{t} .
\end{aligned}
$$


After calculations, we find

$$
L F E_{\sigma}\left\{\sin _{\sigma}\left((a x)^{\sigma}\right)\right\}=\frac{a^{\sigma} v^{3 \sigma}}{1+(a v)^{2 \sigma}} .
$$

6) If $f(x)=\cos _{\sigma}\left((a x)^{\sigma}\right)(0<\sigma \leqslant 1)$, the use of the formula (3.5) gives

$$
\begin{aligned}
L F E_{\sigma}\left\{\cos _{\sigma}\left((a x)^{\sigma}\right)\right\} & =\frac{v^{\sigma}}{\Gamma(1+\sigma)} \int_{0}^{\infty} E_{\sigma}\left(-\frac{x^{\sigma}}{v^{\sigma}}\right) \frac{E_{\sigma}\left(i^{\sigma}(a x)^{\sigma}\right)+E_{\eta}\left(-i^{\sigma}(a x)^{\sigma}\right)}{2}(d x)^{\sigma} \\
& =\frac{1}{2} \frac{v^{\sigma}}{\Gamma(1+\sigma)} \int_{0}^{\infty}\left[E_{\sigma}\left(-\frac{1-(a v)^{\sigma} i^{\sigma}}{v^{\sigma}} x^{\sigma}\right)+E_{\sigma}\left(-\frac{1+(a v)^{\sigma} i^{\sigma}}{v^{\sigma}} x^{\sigma}\right)\right](d x)^{\sigma} \\
& =\frac{v^{\sigma}}{2} \lim _{t \rightarrow \infty}\left[-\frac{v^{\sigma}}{1-(a v)^{\sigma} i^{\sigma}} E_{\sigma}\left(-\frac{1-(a v)^{\sigma} i^{\sigma}}{v^{\sigma}} x^{\sigma}\right)-\frac{v^{\sigma}}{1+(a v)^{\sigma} i^{\sigma}} E_{\sigma}\left(-\frac{1+(a v)^{\sigma} i^{\sigma}}{v^{\sigma}} x^{\sigma}\right)\right]_{0}^{t} .
\end{aligned}
$$

After calculations, we find

$$
L F E_{\sigma}\left\{\cos _{\sigma}\left((a x)^{\sigma}\right)\right\}=\frac{v^{2 \sigma}}{1+(a v)^{2 \sigma}} .
$$

7) If $f(x)=\sinh _{\sigma}\left((a x)^{\sigma}\right)(0<\sigma \leqslant 1)$, we obtain

$$
\begin{aligned}
L F E_{\sigma}\left\{\sinh _{\sigma}\left((a x)^{\sigma}\right)\right\} & =\frac{v^{\sigma}}{\Gamma(1+\sigma)} \int_{0}^{\infty} E_{\sigma}\left(-\frac{x^{\sigma}}{v^{\sigma}}\right) \frac{E_{\sigma}\left((a x)^{\sigma}\right)-E_{\sigma}\left(-(a x)^{\sigma}\right)}{2}(d x)^{\sigma} \\
& =\frac{1}{2} \frac{v^{\sigma}}{\Gamma(1+\sigma)} \int_{0}^{\infty}\left[E_{\sigma}\left(-\frac{1-(a v)^{\sigma}}{v^{\sigma}} x^{\sigma}\right)-E_{\sigma}\left(-\frac{1+(a v)^{\sigma}}{v^{\sigma}} x^{\sigma}\right)\right](d x)^{\sigma} \\
& =\frac{v^{\sigma}}{2} \lim _{t \rightarrow \infty}\left[-\frac{v^{\sigma}}{1-(a v)^{\sigma}} E_{\sigma}\left(-\frac{1-(a v)^{\sigma}}{v^{\sigma}} x^{\sigma}\right)+\frac{v^{\sigma}}{1+(a v)^{\sigma}} E_{\sigma}\left(-\frac{1+(a v)^{\sigma}}{v^{\sigma}} x^{\sigma}\right)\right]_{0}^{t}
\end{aligned}
$$

By performing simple operations, we find

$$
L F E_{\sigma}\left\{\sinh _{\sigma}\left((a x)^{\sigma}\right)\right\}=\frac{a^{\sigma} v^{3 \sigma}}{1-(a v)^{2 \sigma}} .
$$

8) If $f(x)=\cosh _{\sigma}\left((a x)^{\sigma}\right)(0<\sigma \leqslant 1)$, we obtain

$$
\begin{aligned}
\operatorname{LFE}_{\sigma}\left\{\sinh _{\sigma}\left((a x)^{\sigma}\right)\right\} & =\frac{v^{\sigma}}{\Gamma(1+\sigma)} \int_{0}^{\infty} E_{\sigma}\left(-\frac{x^{\sigma}}{v^{\sigma}}\right) \frac{E_{\sigma}\left((a x)^{\sigma}\right)+E_{\sigma}\left(-(a x)^{\sigma}\right)}{2}(d x)^{\sigma} \\
& =\frac{1}{2} \frac{v^{\sigma}}{\Gamma(1+\sigma)} \int_{0}^{\infty}\left[E_{\sigma}\left(-\frac{1-(a v)^{\sigma}}{v^{\sigma}} x^{\sigma}\right)+E_{\sigma}\left(-\frac{1+(a v)^{\sigma}}{v^{\sigma}} x^{\sigma}\right)\right](d x)^{\sigma} \\
& =\frac{v^{\sigma}}{2} \lim _{t \rightarrow \infty}\left[-\frac{v^{\sigma}}{1-(a v)^{\sigma}} E_{\sigma}\left(-\frac{1-(a v)^{\sigma}}{v^{\sigma}} x^{\sigma}\right)-\frac{v^{\sigma}}{1+(a v)^{\sigma}} E_{\sigma}\left(-\frac{1+(a v)^{\sigma}}{v^{\sigma}} x^{\sigma}\right)\right]_{0}^{t} .
\end{aligned}
$$

By performing simple operations, we get

$$
L F E_{\sigma}\left\{\sinh _{\sigma}\left((a x)^{\sigma}\right)\right\}=\frac{v^{2 \sigma}}{1-(a v)^{2 \sigma}} .
$$




\section{Illustrative examples}

Finally, we'll use the local fractional Elzaki transform to solve the local fractional differential equations that have been suggested.

Example 4.1. We consider the local fractional differential equation of order $\sigma$

$$
\frac{d^{\sigma} U(x)}{d x^{\sigma}}-U(x)=1, \quad 0<\sigma \leqslant 1,
$$

with the initial condition $U(0)=0$.

On both sides of (4.1), the local fractional Elzaki transform yields

$$
\frac{1}{v^{\sigma}} \operatorname{LFE} E_{\sigma}\{U(x)\}-v^{\sigma} U(0)-L F E_{\sigma}\{U(x)\}=L F E_{\sigma}\{1\}
$$

Then

$$
\left(\frac{1}{v^{\sigma}}-1\right) \operatorname{LFE} E_{\sigma}\{U(x)\}=v^{2 \sigma}
$$

Which give

$$
\begin{aligned}
\operatorname{LFE}_{\sigma}\{U(x)\} & =\frac{v^{3 \sigma}}{1-v^{\sigma}} \\
& =\frac{v^{2 \sigma}}{1-v^{\sigma}}-v^{2 \sigma} .
\end{aligned}
$$

We get (4.4) by doing the inverse transformation on both sides

$$
U(x)=E_{\sigma}\left(-x^{\sigma}\right)-1,
$$

which is the exact solution of the equation (4.1). $E_{\sigma}$ is the Mittag-Leffler function.

Example 4.2. Next, we consider the following local fractional differential equation of order $\sigma,(0<\sigma \leqslant 1)$

$$
\frac{d^{\sigma} U(x)}{d x^{\sigma}}-2 U(x)=4
$$

with the initial condition

$$
U(0)=0
$$

Taking local fractional Elzaki transform on both sides of (4.6), we have

$$
\frac{1}{v^{\sigma}} \operatorname{LFE}\{U(x)\}-2 L F E_{\sigma}\{U(x)\}=4 v^{2 \sigma} .
$$


By following the same steps as the previous example, we obtain

$$
L F E_{\sigma}\{U(x)\}=-2 v^{2 \sigma}+2 \frac{v^{2 \sigma}}{1-2 v^{\sigma}} .
$$

Take the inverse transformation on both sides of (4.9), we get

$$
U(x)=2 E_{\sigma}\left(2 x^{\sigma}\right)-2
$$

The result (4.10) represents the exact solution of (4.6) - (4.7).

Example 4.3. We consider the local fractional differential equation of order $2 \sigma,(0<\sigma \leqslant 1)$

$$
\frac{d^{2 \sigma} U(x)}{d x^{2 \sigma}}+U(x)=-\frac{x^{\sigma}}{\Gamma(1+\sigma)}
$$

with to the initial conditions

$$
U(0)=0, U^{(\sigma)}(0)=0
$$

Taking local fractional Elzaki transform on both sides of (4.11), we get

$$
\frac{1}{v^{2 \sigma}} L_{F} E_{\sigma}\{U(x)\}+L F E_{\sigma}\{U(x)\}=-v^{3 \sigma} .
$$

We get the same result as the previous example by following the same steps

$$
\operatorname{LFE}_{\sigma}\{U(x)\}=-v^{3 \sigma}+\frac{v^{3 \sigma}}{1+v^{2 \sigma}} .
$$

Take the inverse transformation on both sides of (4.14), we get

$$
U(x)=\sin _{\sigma}\left(x^{\sigma}\right)-\frac{x^{\sigma}}{\Gamma(1+\sigma)},
$$

so this result (4.15) represents the exact solution to the equation (4.11) - (4.12).

\section{Conclusion}

The idea that we presented in this paper is based on combining the Elzaki transform with the local fractional derivative, where we present some important results of this combination which called: Local fractional Elzaki transform of the Mittag-Leffler function, the hyperbolic sine and the hyperbolic cosine in fractal space and also we provide its properties of some non-differentiable functions were presented. This method was used to solve several linear local fractional differential equations, where we have seen that the nondifferential solutions are precise and these results lead us to say that the local fractional Elzaki transform is powerful and effective in solving this type of equation, and thus can be applied to other linear local fractional partial differential equations with variable coefficients, to the linear systems of differential equations and to the other linear problems in Cantor sets. 


\section{Author Contributions}

All authors contributed equally to this work. They all read and approved the final version of the manuscript.

\section{Conflicts of Interest}

The authors declare no conflict of interest.

\section{Acknowledgement}

We thank Dr. Ali YAKAR for technical support. The authors would like to thank editor and the reviewers for the reading carefully of the manuscript and the detail comments and valuable suggestions which has helped us to improve this paper.

\section{References}

\section{References}

[1] M. R. Spiegel, Theory and problems of Laplace transform, McGraw Hill, New York, 1965.

[2] S. Benzoni, Analyse de Fourier, Universite de Lyon, Lyon 1, Camille Jordan Institute, Saint-Étienne, 2011.

[3] N. T. Negero, Zero-Order Hankel Transform Method for Partial Differential Equations, International Journal of Modern Science and Engineering Technology, 3(10), (2016) 24-36.

[4] D. Lomen, Application of the Mellin Transforin to Boundary Value Problems, Proceedings of the Iowa Academy of Science, 69(1), (1962) 436-442.

[5] G. K. Watugala, Sumudu transform: a new integral transform to solve differentia lequations and control engineering problems, International Journal of Mathematical Education in Science and Technology, 24(1), (1993) 35-43.

[6] Z. H. Khan, W. A. Khan, N-transform properties and applications, NUST Journal of Engineering Sciences, 1, (2008) 127-133.

[7] T. M. Elzaki, The New Integral Transform "ELzaki Transform", Global Journal of Pure and Applied Mathematics, 7(1), (2011) 57-64.

[8] H. J. Kim, The time shifting theorem and the convolution for Elzaki transform, Global Journal of Pure and Applied Mathematics, 87, (2013) 261-271.

[9] A. Devi, P. Roy, V. Gill, Solution of ordinary differential equations with variable coefficients using Elzaki transform, Asian Journal of Applied Science and Technology, 1, (2017) 186-194.

[10] A. Kalavathi, T. Kohila, L. M. Upadhyaya, On the degenerate Elzaki transform, Bulletin of Pure and Applied Sciences Section -E- Mathematics \& Statistics, 40E(1), (2021) 99-107.

[11] K. S. Aboodh, The new integrale transform "Aboodh transform", Global Journal of Pure and Applied Mathematics, 9(1), (2013) 35-43.

[12] Z. U. Zafar, ZZ Transform Method, International Journal of Advanced Engineering and Global Technoloy, 4(1), (2016) 1605-1611. 
[13] S. Maitama, W. Zhao, New Integral Transform: Shehu Transform a Generalization of Sumudu and Laplace Transform for Solving differential equations, International Journal of Analysis and Applications, 17(2), (2019) 167-190.

[14] M. S. Archana, V. J. Pratibha, Elzaki Transform: A Solution of Differential Equations, International Journal of Scientific Engineering \& Technology Research, 4(4), (2015) 1006-1008.

[15] P. P. Chopade, S. B. Devi, Applications of Elzaki Transform to Ordinary Differential Equations and Partial Differential Equations, International Journal Advanced Research in Computer Science Software Engineering, 5(3), (2015) 38-41.

[16] M. Eslaminasab, S. Abbasbandy, Study on usage of Elzaki transform for the ordinary differential equations with non-constant coefficients, International Journal of Industrial Mathematics, 7(3), (2015) 277281.

[17] T. M. Elzaki, S. M. Ezaki, On the ELzaki Transform and Ordinary Differential Equation with Variable Coefficients, Advances in Theoretical and Applied Mathematics, 6(1), (2011) 41-46.

[18] T. M. Elzaki, S. M. Ezaki, On the ELzaki Transform and Higher Order Ordinary Differential Equations, Advances in Theoretical and Applied Mathematics, 6(1), (2011) 107-113.

[19] T. M. Elzaki, S. M. Ezaki, Solution of Integro-Differential Equations by Using ELzaki Transform, Global Journal of Mathematical Sciences: Theory \& Practical , 3(1), (2011) 1-11.

[20] M. M. A. Mahgob, Elzaki Transform and a Bulge Function on Volterra Integral Equations of the Second Kind, IOSR Journal of Mathematics, 11(2), (2012) 68-70.

[21] M. M. A. Mahgob, T.M. Elzaki, Solution of Partial Integro-Differential Equations by Elzaki Transform Method, Applied Mathematical Sciences, 9(6), (2015) 295-303.

[22] M. M. A. Mahgob, T. M. Elzaki, Elzaki Transform and Integro-Differential Equation with a Bulge Function, IOSR Journal of Mathematics, 11(2), (2015) 25-28.

[23] P. G. Bhadane, V. H. Pradhan, S. V. Desale, Elzaki Transform Solution of One Dimensional Ground Water Recharge through Spreading, International Journal of Engineering Research and Applications, 3(6), (2013) 1607-1610.

[24] T. M. Elzaki, E. M. A. Hilal, Analytical Solution for Telegraph Equation by Modified of Sumudu Transform "Elzaki Transform", Mathematical Theory and Modeling, 2(4), (2012) 104-111.

[25] T. M. Elzaki, S. M. Ezaki, On the ELzaki Transform and System of Partial Differential Equations, Advances in Theoretical and Applied Mathematics, 6(1), (2011) 115-123.

[26] D. Ziane, M. Hamdi Cherif, Resolution of Nonlinear Partial Differential Equations by Elzaki Transform Decomposition Method, Journal of Approximation Theory and Applied Mathematics, 5, (2015) 17-30.

[27] T. M. Elzaki, S. M. Elzaki, Applications of New Transform "ELzaki Transform" to Partial Differential Equations, Global Journal of Pure and Applied Mathematics, 7(1), (2011) 65-70.

[28] A. Devi, M. Jakhar, Analytic solution of fractional order differential equation arising in RLC electrical circuit, Malaya Journal of Matematik, 8(2), (2020) 421-426. 
[29] D. Ziane, Application of Homotopy Analysis Method Combined with Elzaki Transform for Fractional Porous Medium Equation, Journal of Approximation Theory and Applied Mathematics, 6, (2019) 1-19.

[30] D. Ziane, Elzaki transform and the decomposition method for nonlinear fractional partial differential equations, International Journal of Open Problems in Computer Science and Mathematics, 9(4), (2016) 25-39.

[31] D. Ziane, M. Hamdi Cherif, K. Belghaba, Fractional higher dimensional initial boundary value problems via variational iteration method coupled with Elzaki transform, Nonlinear Studies, 24(4), (2017) 1-17.

[32] D. Ziane, T. M. Elzaki, M. Hamdi Cherif, Elzaki transform combined with variational iteration method for partial differential equations of fractional order, Fundamental Journal of Mathematics and Applications, 1(1), (2018) 102-108.

[33] H. M. Srivastava, A. K. Golmankhaneh, D. Baleanu, X. J. Yang, Local Fractional Sumudu Transform with Application to IVPs on Cantor Sets, Abstract and Applied Analysis, 2014, (2014) Article ID: 176395, 1-7.

[34] X. J. Yang, Fractional Functional Analysis and Its Applications, Asian Academic, Hong Kong, 2011.

[35] X. J. Yang, Local Fractional Calculus and Its Applications, World Scientific Publishing, New York, 2012.

[36] J. H. He, Asymptotic Methods for Solitary Solutions and Compactons, Abstract and Applied Analysis, 2012, (2012) Article ID: 916793, 1-130.

[37] C. G. Zhao, A. M. Yang, H. Jafari, A. Haghbin, The Yang-Laplace Transform for Solving the IVPs with Local Fractional Derivative, Abstract and Applied Analysis, 2014, Article ID: 386459, (2014) 1-5.

[38] X. J. Yang, L. Li, R. Yang, Problems of local fractional definite integral of the one-variable nondifferentiable function, World Science and Technology R\&D, 31(4), (2009) 722-724.

[39] J. Ahmad, S. T. Mohyud-Din, H. M. Srivastava, X-J. Yang, Analytic solutions of the Helmholtz and Laplace equations by using local fractional derivative operators, Waves, Wavelets and Fractals - Advanced Analysis, 1, (2015) 22-26.

[40] X. J. Yang, Generalized Sampling Theorem for Fractal Signals, Advances in Digital Multimedia, 1(2), (2012) 88-92.

[41] G. Jumarie, Table of some basic fractional calculus formulae derived from a modified Riemann-Liouville derivative for non-differentiable functions, Applied Mathematics Letters, 22, (2009) 378-385. 